\title{
Pain clinics, a new role for psychiatrists
}

\section{Results of a questionnaire survey of liaison psychiatrist involvement in pain clinics in the Greater London area}

\section{AIMS AND METHOD}

The aim of this study was to find out the extent of psychiatric involvement in pain clinics and also clarify the treatment approaches taken for the significant psychiatric comorbidity reported in these clinics. A questionnaire survey was conducted, by a psychiatrist and an anaesthetist, of the 31 pain clinics in the Greater London area.

\section{RESULTS}

Psychiatric involvement in the management of patients with chronic pain is extremely limited, despite the extensive psychiatric morbidity associated with these patients (response rate, $74 \%$ ). The majority of surveyed pain clinics wish to provide a multi-disciplinary approach to these patients, utilising the skills of a psychiatrist for both assessment and management. Only a small minority of pain clinics, however, have sufficient access to a liaison psychiatry service that could provide the broad multidisciplinary approach and the psychiatric treatment options that they believe chronic pain patients require.

\section{IMPLICATIONS}

The involvement of psychiatrists in pain clinics is very limited. Increased involvement is desired and would likely lead to an improved profile of pain clinics, a more realistic and comprehensive treatment approach and, in turn, enhanced patient care.
The treatment of chronic pain is complex and, probably more than any other area in medicine, needs a multidisciplinary approach. The pain service is currently regarded as an anaesthetic sub-speciality and the director of the service is usually a consultant anaesthetist who has an interest in chronic pain. An editorial in Anaesthesia stressed the need for increased involvement of liaison psychiatrists in pain clinics, given the upsurge of interest in the role of liaison psychiatry in the management of medical conditions in general and that many pain patients have significant psychological and psychiatric difficulties (Dolin \& Stephens, 1998). We felt that now is an appropriate time to investigate the staff profile of pain clinics, especially the current involvement of the liaison psychiatrist in the pain clinic service. We also attempted to explore the chronic pain service attitude towards a multidisciplinary approach to the management of pain.

The staff composition of pain clinics generally varies according to local resources and interest. The pain clinic may have the benefit of input from a number of specialities, including anaesthetists, psychologists, acupuncturists, physiotherapists and psychiatrists. Frequently, however, the clinic is run by the anaesthetist on his or her own, with limited input from other professionals.

There are a variety of recognised psychiatric disorders found in chronic pain patients, the most obvious being depression (Romano \& Turner, 1985), but substance misuse and post-traumatic stress disorder are also commonly seen (Geisser et al, 1996). Pain patients with psychiatric morbidity can be very difficult to manage and can benefit from the specialist skill of a psychiatrist (DeGood \& Kiernan, 1996). Perhaps the most challenging pain patients are those for whom no sufficient physical diagnosis can be made. Many of these patients reach the pain clinic after years of failed treatments, further adding to management difficulties and requiring the specialist skills of a psychiatrist (Dolin \& Stephens, 1998).

Diagnosis and treatment of the significant psychiatric morbidity associated with chronic pain may benefit from the multi-disciplinary approach of a psychiatrist. As to how a psychiatrist would work in a pain clinic, Dolin and Stephens (1998) advocate a joint clinic according to a multidisciplinary assessment model. Joint assessment of the pain patient by an anaes thetist and a psychiatrist has the advantage of being an unspoken rejection of the mind-body divide and is a natural acceptance of the nature of pain as having both physical and psychological components.

The obvious value of psychiatric involvement in pain clinics has been emphasised but unfortunately liaison psychiatry is still a scarce resource (Mayou et al, 1990). To clarify the situation we decided to inquire into the extent of liaison psychiatry involvement in pain clinics in the Greater London area.

\section{Method}

The aim of this survey was to examine the extent of multi-disciplinary input and, in particular, psychiatric input into the pain management services in the Greater London area and to estimate the perceived benefit of the multidisciplinary approach to chronic pain.

All 31 pain clinics in the Greater London area were sent a specially designed questionnaire, two pages long, with a covering letter explaining the purpose of the survey. The clinics were identified by The Pain Society of Great Britain. The letter stated that the authors, an anaesthetist and a psychiatrist, were interested in the staff profile of pain clinics and how comorbid psychiatric conditions were addressed. If a questionnaire 
was not returned within 6 weeks a second letter and questionnaire were sent.

original

papers

\section{Results}

Completed questionnaires were returned by 23 pain clinics, that is, $74 \%$ of the total sample $(n=31)$.

Pain clinics in London have, on average, access to the skills of three consultants, but this state of affairs varies significantly; some pain clinics are single-handed operations while others are favoured with involvement of up to 10 consultants. The overwhelming majority of pain clinics have an anaesthetist on the staff (95\%); in fact the anaesthetist is usually the person in charge, pain management being generally regarded as a sub-speciality of anaesthetics.

Input from other professionals for the management of pain patients varied considerably in our study, with $52 \%$ of pain clinics having access to a pain nurse, $39 \%$ to a physiotherapist and $22 \%$ benefiting from the skills of an occupational therapist. Of responding pain clinics, 56\% report a clinical psychologist as having an involvement with the clinic, the psychologist being referred selected patients (whom they may see in a separate room during clinic time, as occurs at the authors' clinic).

Despite the wide range of recognised psychiatric comorbidity associated with pain patients, only $30 \%$ of pain clinics reported having a psychiatrist on the staff. When present, psychiatrists contributed, on average, 2.5 sessions per week to the pain clinics.

It is a rare occurrence for a psychiatrist and a pain clinician to see the chronic pain patient together (as is standard in the authors' clinic) and when they do it only occurs with a few highly selected patients. Less than $25 \%$ of pain clinics offer a 'combined assessment' of the pain patient by the pain consultant and a psychiatrist. Unfortunately, where combined assessment is carried out it is used sparingly, on selected patients only. Only two of the 23 responding pain clinics offer combined assessment by a pain clinician and a psychiatrist to all of their patients.

When it comes to the management of psychiatric morbidity, $56 \%$ of pain clinics would favour, where possible, a combined treatment approach in dealing with depression, using both cognitive-behavioural techniques, including cognitive-behavioural therapy (CBT), and antidepressant medication. In dealing with comorbid depression only $4 \%$ of surveyed pain clinics would use CBT alone and only $13 \%$ would use antidepressants alone to treat depression in the context of chronic pain.

While $78 \%$ of pain clinics have team meetings to discuss management issues, only $50 \%$ felt that they were able to incorporate some elements of a multidisciplinary approach into the assessment and treatment of chronic pain patients and their varied psychiatric difficulties. One particularly disadvantaged group was clinics that had access to neither a clinical psychologist nor a psychiatrist.

\section{Comment}

The psychiatrist brings many advantages to the pain clinic: the ability to make diagnoses and also the ability to take a broad view of management that incorporates both pharmacological and psychological treatments. Furthermore, liaison psychiatrists bring with them the ability to place individuals in a social context and recognise the importance of family factors as they impinge on chronic pain.

Refined prescribing skills acquired by the psychiatrist are also of importance in the treatment of the anxiety, substance misuse and depressive disorders often associated with the chronic pain patient. Cognitivebehavioural skills, with an emphasis on lifestyle changes, can often be of significant advantage in the pain clinic. Our survey, however, shows that this expertise, frequently associated with the liaison psychiatrist, is often inaccessible to the pain clinician.

Joint assessment by the pain clinician and psychiatrist promotes a holistic approach to assessment and management and is a rejection of the mind-body dichotomy. The joint assessment involves an acceptance of the multi-factorial nature of pain and promotes communication and understanding between disciplines. Our survey suggests that joint assessment in the pain clinic is rare and that when it does occur it is very selective, offered to a few patients only. Furthermore, it is likely that our survey overestimates psychiatric input to pain clinics because it was carried out in Greater London, where quite a lot of sessional time for liaison psychiatry comes from academic psychiatrists.

The shortage of and need for improved liaison psychiatry in the UK has been emphasised in a recent joint report of the Royal Colleges of Physicians and Psychiatrists (1995). Pain clinics wish to offer a multidisciplinary approach to their patients but our survey supports the view that there are still too few pain clinics that have the opportunity to work with liaison psychiatrists and so offer this preferred option. Increased availability of liaison psychiatrists within the pain clinic service will meet a significant need, may improve the profile of these clinics and should also enhance patient care.

\section{References}

DEGOOD, D. E. \& KIERNAN, B. (1996) Perception of fault in patients with chronic pain. Pain, 64, 153-159.

DOLIN, S. \& STEPHENS, J. (1998) Pain clinics and liaison psychiatry. Anaesthesia, 53, 317-319.

GEISSER, M., ROTH, R., BAEHMAN, J. et al (1996) The relationship between symptoms of post traumatic stress disorder and pain, affective disturbance and disability in accident and nonaccidentrelated pain. Pain, 66, 207-214.

MAYOU, R., ANDERSON, H. FEINMANN, C., et al (1990) The present state of consultation and liaison psychiatry. Psychiatric Bulletin, 14 321-325.

ROMANO, J. \& TURNER, J. (1985) Chronic pain and depression: does the evidence support a relationship? Psychology Bulletin, 97, 18-32.

ROYAL COLLEGE OF PHYSICIANS \& ROYAL COLLEGE OF PSYCHIATRISTS (1995) The Psychological Care of Medical Patients. Recognition of Need and Service Provision. Council Report CR35. London. Royal College of Physicians \& Royal College of Psychiatrists.
*John Wallace Clinical Psychologist and Specialist Research Psychiatrist, Social, Genetic and Developmental Psychiatry Research Centre, The Institute of Psychiatry, Maudsley Hospital, 111 Denmark Hill, London SE5 8AF, Gnanie Panch Consultant Anaesthetist, Department of Anaesthetics, Whittington Hospital, Highgate Hill, London N19 5NF 